\author{
F. Cipollone $\cdot$ F. Chiarelli $\cdot$ G. Davì $\cdot$ C. Ferri $\cdot$ \\ G. Desideri · M. Fazia - A. Iezzi · F. Santilli $\cdot$ B. Pini • \\ C. Cuccurullo $\cdot$ S. Tumini $\cdot$ A. Del Ponte $\cdot$ A. Santucci $\cdot$ \\ F. Cuccurullo $\cdot$ A. Mezzetti
}

\title{
Enhanced soluble CD40 ligand contributes to endothelial cell dysfunction in vitro and monocyte activation in patients with diabetes mellitus: effect of improved metabolic control
}

Received: 6 August 2004 / Accepted: 9 February 2005 / Published online: 3 May 2005

(C) Springer-Verlag 2005

\begin{abstract}
Aims/hypothesis: Inflammation plays a pathogenic role in the development of accelerated atherosclerosis in diabetes. Soluble CD40 ligand (sCD40L) is enhanced in diabetes; however, the molecular mechanisms linking SCD40L to accelerated atherosclerosis in diabetes are still unclear. We tested the hypothesis that $\mathrm{SCD} 40 \mathrm{~L}$ may be involved in the vascular complications in diabetes and exerts its effect by triggering inflammatory reactions on mononuclear and endothelial cells (ECs). Methods: We studied 70 patients, 40 with type 2 and 30 with type 1 diabetes, with a history or physical examination negative for cardiovascular disease, and 40 non-diabetic and 30 healthy subjects, matched with the type 2 and type 1 diabetic patients, respectively. Plasma and serum SCD40L, and plasma soluble intercellular adhesion molecule-1, soluble vascular cell adhesion molecule-1, E-selectin and monocyte chemo-attrac-
\end{abstract}

F. Cipollone $\cdot$ F. Chiarelli · G. Davì $\cdot$ M. Fazia $\cdot$ A. Iezzi

F. Santilli - B. Pini - C. Cuccurullo - S. Tumini - A. Del Ponte

F. Cuccurullo $\cdot$ A. Mezzetti

Atherosclerosis Prevention Center,

'G. d'Annunzio' University of Chieti,

Chieti, Italy

F. Cipollone · F. Chiarelli · G. Davì · M. Fazia · A. Iezzi .

F. Santilli - B. Pini - C. Cuccurullo - S. Tumini - A. Del Ponte ·

F. Cuccurullo - A. Mezzetti

Clinical Research Center,

'G. d'Annunzio' University Foundation,

Chieti, Italy

C. Ferri · G. Desideri - A. Santucci

School of Medicine, University of L'Aquila,

L'Aquila, Italy

F. Cipollone $(\bowtie)$

Centro Regionale per la Prevenzione dell'

Aterosclerosi e Centro di Ricerca Clinica,

Centro per lo Studio dell' Invecchiamento (Ce.S.I),

Via Colle dell'Ara,

66013 Chieti, Italy

e-mail: fcipollone@unich.it

Tel: +39-0871-541360

Fax: +39-0871-541491 tant protein-1 (MCP-1) were measured. Adhesion molecules and MCP-1 release, the ability to repair an injury in $\mathrm{ECs}$, and $\mathrm{O}_{2}^{-}$generation in monocytes were analysed in vitro after stimulation with serum from patients or controls. Results: Type 2 and type 1 diabetic patients had significantly higher sCD40L levels than controls. Furthermore, high SCD40L was associated with in vitro adhesion molecules and MCP-1 release, impaired migration in ECs and enhanced $\mathrm{O}_{2}^{-}$generation in monocytes. Improved metabolic control was associated with a reduction of plasma $\mathrm{SCD} 40 \mathrm{~L}$ by $37.5 \%$ in 12 type 1 diabetic patients. Furthermore, elevated $\mathrm{sCD} 40 \mathrm{~L}$ in diabetic patients was significantly correlated with $\mathrm{HbA}_{1} \mathrm{c}$ levels. Conclusions/interpretation: Upregulation of $\mathrm{sCD} 40 \mathrm{~L}$ as a consequence of persistent hyperglycaemia in diabetic patients results in EC activation and monocyte recruitment to the arterial wall, possibly contributing to accelerated atherosclerosis development in diabetes.

Keywords Atherosclerosis - Diabetes mellitus · Endothelium · Inflammation · Soluble CD40 ligand

Abbreviations EC: Endothelial cell $\cdot \mathrm{MCP}-1$ : Monocyte chemo-attractant protein-1 $\cdot$ NF- $\mathrm{KB}$ : Nuclear factor- $\kappa \mathrm{B}$. NO: Nitric oxide - PKC: Protein kinase C - ROS: Reactive oxygen species - sCD40L: Soluble CD40 ligand ·

sE-selectin: Soluble E-selectin · sICAM-1: Soluble intercellular adhesion molecule-1 - sVCAM-1: Soluble vascular cell adhesion molecule-1 - TZD:

Thiazolidinedione

\section{Introduction}

Diabetes mellitus is a major source of morbidity in developed countries and, among its comorbid conditions, atherosclerosis is perhaps the most important.

Diabetes is associated with a variety of metabolic abnormalities, the principal of which is hyperglycaemia. Interestingly, prospective data have suggested a link between a 
marker for chronic average glucose levels $\left(\mathrm{HbA}_{1} \mathrm{c}\right)$ and cardiovascular morbidity and mortality [1]. However, there is considerable controversy with respect to the precise mechanism by which hyperglycaemia may contribute to the rapid development of coronary artery disease in diabetes.

CD40 ligand (CD40L, also known as CD154), a transmembrane protein structurally related to TNF- $\alpha$, was originally identified on $\mathrm{CD} 4+\mathrm{T}$ cells, but it was recently found also on activated platelets [2]. Both membrane-bound and soluble forms of this ligand may interact with CD40, which is constitutively expressed on B cells, macrophages, endothelial cells (ECs), and vascular smooth muscle cells, resulting in various inflammatory responses [3]. Recently the presence of T cells expressing CD40L within atherosclerotic lesions in humans has been reported [3], and the ability of gene disruption or anti-CD40L antibody treatment to limit the evolution and the instability of atherosclerotic plaque has been described [4]. Increased soluble CD40L (sCD40L) has been observed in unstable angina [5] and hypercholesterolaemia [6], and it has been recently reported that circulating $\mathrm{sCD} 40 \mathrm{~L}$ has a strong independent prognostic value among apparently healthy individuals [7] and patients with acute coronary syndromes $[8,9]$.

Thus, it is now generally accepted that CD40L-CD40 interaction is an initial event in atherothrombosis, leading in turn to the activation of several pro-inflammatory mediators [10]. In fact, CD40L may promote the expression of vessel-remodelling metalloproteinases and induce pro-coagulant activity in vascular cells, and the absence of CD40L affects the stability of arterial thrombi and delays arterial occlusion in vivo [11]. Moreover, ligation of CD40 on ECs inhibits cell migration after injury by increasing production of reactive oxygen species (ROS) [12], and upregulates monocyte chemo-attractant protein-1 (MCP-1), soluble E-selectin (sE-selectin), soluble vascular cell adhesion molecule-1 (sVCAM-1) and soluble intercellular adhesion molecule-1 (sICAM-1) [2, 13], all molecules that have been associated with atherothrombosis by mediating the recruitment and adhesion of monocytes and lymphocytes to the injured vessel wall $[14,15]$.

Worth noting is the recent demonstration of enhanced SCD40L in diabetic patients $[16,17]$, and the ability of a short-term treatment with thiazolidinediones (TZDs) to reduce it $[16,17]$, as a consequence of the anti-inflammatory effects of TZD [17]. However, some points regarding the relationship between $\mathrm{SCD} 40 \mathrm{~L}$ and diabetes are still unclear. First of all, the relationship between enhanced SCD40L and endothelial and monocyte function in diabetes has not yet been investigated. Second, no studies have yet demonstrated whether long-term intensive glycaemic control, as achieved by conventional antidiabetic therapy, may also diminish circulating sCD40L.

Thus, in the present study, we set out to investigate the possible role of $\mathrm{SCD} 40 \mathrm{~L}$ in vascular dysfunction in diabetes. In particular, we tested the hypothesis that SCD40L exerts its detrimental effect by triggering a complex group of inflammatory reactions both in vascular ECs and in circulating monocytes/macrophages. Finally, we analysed the effect of intensive glycaemic control on SCD40L levels in a 5 -year study on 12 type 1 diabetic patients.

\section{Subjects and methods}

\section{Subjects}

Seventy patients with diabetes mellitus were studied. Forty had type 2 diabetes and 30 had type 1 diabetes, defined in accordance with the criteria of the American Diabetes Association [18]. None of the 70 patients had a history or physical examination positive for evidence of micro- or macrovascular complications. Forty non-diabetic subjects and 30 healthy subjects, age-, gender- and risk factor-matched with the type 2 and type 1 patients, respectively, were also studied. The baseline characteristics of patients and control subjects are detailed in Table 1. All the patients had stably achieved the therapeutic goal for both hypertension and hypercholesterolaemia (defined according to the JNC VI and ATP III guidelines, respectively). The study was approved by local ethics review committees and reviewed by the IRB of the G. d'Annunzio University Foundation. Written informed consent was obtained from each patient.

\section{Study design}

In the first study, a cross-sectional comparison of baseline sCD40L, sICAM-1, sVCAM-1, sE-selectin and MCP-1 was performed between patients and control subjects.

To assess the potential influence of improved metabolic control on sCD40L generation, a second study was performed in 12 of the 30 patients with type 1 diabetes. At the time of the cross-sectional study, these 12 type 1 diabetic patients were in poor metabolic control $\left(\mathrm{HbA}_{1} \mathrm{c}>8 \%\right)$ despite taking insulin for months. These patients were examined every 3 months over a 5-year period for clinical and blood glucose monitoring, and intensive insulin therapy was adjusted to achieve improved metabolic control. No patient experienced a hypoglycaemic reaction during the study period. Blood samples were obtained before and at the end of this intensive monitoring and treatment programme for determination of fasting glucose, $\mathrm{HbA}_{1} \mathrm{c}$ levels and sCD40L biosynthesis.

\section{Laboratory procedures}

Blood samples were collected in a fasting condition and immediately centrifuged at $4^{\circ} \mathrm{C}$. Fasting plasma glucose was measured by the glucose oxidase method. HbA1c concentrations were measured by HPLC (Diamat Analyzer; BioRad, Richmond, CA, USA). The normal range was 4.2$6.0 \%$, with an interassay CV of $3 \%$. All the patients had at least three determinations per year and three determinations in the last month of the 5-year treatment period, and the mean of these was used for statistical analysis. 
Table 1 Baseline clinical features of type 1 and 2 diabetic patients and matched nondiabetic controls
Data are means $\pm \mathrm{SD}$ or absolute values

\begin{tabular}{|c|c|c|c|c|}
\hline & Type $2(n=40)$ & Controls $(n=40)$ & Type $1(n=30)$ & Controls $(n=30)$ \\
\hline $\operatorname{Sex}(F / M)$ & $22 / 18$ & $22 / 18$ & $15 / 15$ & $15 / 15$ \\
\hline Age (years) & $65 \pm 10$ & $67 \pm 8$ & $20 \pm 3.3$ & $21 \pm 2.8$ \\
\hline BMI $\left(\mathrm{kg} / \mathrm{m}^{2}\right)$ & $31.4 \pm 6$ & $30.2 \pm 4$ & $24.2 \pm 4.5$ & $22.4 \pm 3$ \\
\hline Duration of diabetes (years) & $11.3 \pm 10.8$ & 0 & $13.7 \pm 3.7$ & 0 \\
\hline Fasting blood glucose (mg/dl) & $182.8 \pm 67.3$ & $91.5 \pm 7$ & $187.4 \pm 84.7$ & $78.4 \pm 8$ \\
\hline $\mathrm{HbA}_{1} \mathrm{c}(\%)$ & $7.6 \pm 2.3$ & $4.4 \pm 0.8$ & $8.87 \pm 1.3$ & $4.2 \pm 1.1$ \\
\hline $\begin{array}{l}\text { Diagnosis of } \\
\text { hypercholesterolaemia }(n)\end{array}$ & 10 & 9 & 0 & 0 \\
\hline $\begin{array}{l}\text { LDL cholesterol at } \\
\text { enlistment }(\mathrm{mg} / \mathrm{dl})\end{array}$ & $91 \pm 4$ & $90 \pm 5$ & $89 \pm 5$ & $93 \pm 3$ \\
\hline Smoking $(n)$ & 4 & 4 & 0 & 0 \\
\hline Hypertension $(n)$ & 28 & 27 & 0 & 0 \\
\hline $\begin{array}{l}\text { Systolic and Diastolic blood } \\
\text { pressure at } \\
\text { enlistment }(\mathrm{mm} \mathrm{Hg})\end{array}$ & $122 \pm 4 / 76 \pm 6$ & $124 \pm 3 / 79 \pm 3$ & $117 \pm 5 / 71 \pm 4$ & $119 \pm 4 / 72 \pm 3$ \\
\hline $\begin{array}{l}\text { Family history of cardiovascular } \\
\text { disease }(n)\end{array}$ & 12 & 11 & 0 & 0 \\
\hline \multicolumn{5}{|l|}{ Treatment $(n)$ with: } \\
\hline Angiotensin receptor blockers & 8 & 9 & 0 & 0 \\
\hline ACE-inhibitors & 20 & 19 & 0 & 0 \\
\hline$\beta$-blockers & 1 & 1 & 0 & 0 \\
\hline$\alpha$-blockers & 5 & 4 & 0 & 0 \\
\hline $\mathrm{Ca}^{2+}$ channel blockers & 7 & 8 & 0 & 0 \\
\hline Diuretics & 12 & 10 & 0 & 0 \\
\hline Statins & 10 & 9 & 0 & 0 \\
\hline
\end{tabular}

Enzyme immunoassays

Serum and plasma samples were collected from all patients after a 12 -h fast, and immediately stored at $-80^{\circ} \mathrm{C}$ until analysis. Specific enzyme immunoassays for sCD40L, sICAM-1, sVCAM-1 and sE-selectin (Bender Medsystems, Vienna, Austria), and MCP-1 (BioSource, Camarillo, CA, USA) were used in triplicate as previously described [14], to increase the data accuracy. At our laboratory, the intraassay and interassay $\mathrm{CV}$ values were $<6 \%$. Finally, all analyses were performed by an operator blinded to the patients' characteristics.

\section{sCD40L-dependent EC activation}

HUVECs were harvested from fresh human umbilical cord veins and cultured as previously described [19]. After 3 and $18 \mathrm{~h}$ in culture, sICAM-1, sVCAM-1, sE-selectin and MCP-1 generation was measured by ELISA according to previously described and validated methods [14].

\section{EC migration}

HUVECs were isolated and cultured, and cell migration was analysed according to previously described and validated methods [19]. Cell viability was evaluated by the MTS [3-(4,5-dimethylthiazol-2-yl)-5-(3-carboxymethoxyphenyl)-2-(4-sulphophenyl)-2H-tetrazolium, inner salt] col- orimetric assay (Promega Corp., Madison, WI, USA). Some experiments were repeated in the presence of synthetic sCD40L $(10 \mathrm{ng} / \mathrm{ml}$ and $1-10 \mu \mathrm{g} / \mathrm{ml}$ ) (Alexis Biochemicals Corporation, San Diego, CA, USA) and anti-human sCD40L blocking monoclonal antibody $(10$ and $50 \mu \mathrm{g} / \mathrm{ml})$ (Alexis). Incubation with isotype-matched control immunoglobulin served as negative control. Assays were performed in triplicate and repeated at least twice.

\section{sCD40L-dependent monocyte activation}

The isolation and culture of peripheral monocytes from five healthy blood donors and the analysis of $\mathrm{O}_{2}^{-}$generation was assessed as previously described [9, 14]. Synthetic sCD40L $(10 \mathrm{ng} / \mathrm{ml}$ and $1-10 \mu \mathrm{g} / \mathrm{ml})$, anti-human blocking monoclonal antibody for CD40L $(10 \mu \mathrm{g} / \mathrm{ml})$ (Alexis), or control mouse $\operatorname{IgG}_{1}(50 \mu \mathrm{g} / \mathrm{ml})$ were added to some cell cultures. Furthermore, in some experiments chelerythrine was also added to test the hypothesis that protein kinase $\mathrm{C}$ (PKC) plays an important role in vascular superoxide production in diabetes.

\section{Statistical analysis}

The data were analysed by non-parametric methods to avoid assumptions about the distribution of the measured variables. ANOVA was performed with the Kruskal-Wallis method. Subsequent pairwise comparisons were made with 
the Mann-Whitney $U$-test. The differences between baseline and post-treatment values were analysed with the Wilcoxon signed rank test. Correlations between biochemical parameters were assessed by the Spearman test. The data are expressed as means \pm SD. Statistical significance was considered to be indicated by $p<0.05$. All calculations were performed using the computer program SPSS 11.0.1.

\section{Results}

Circulating levels of sCD40L

Plasma sCD40L was significantly higher in patients with type 2 diabetes than in matched non-diabetic subjects (9.2 \pm 3.4 vs $1.53 \pm 0.45 \mathrm{ng} / \mathrm{ml}$, means $\pm \mathrm{SD}, n=40 ; p<0.003$; Fig. $1 \mathrm{a}$ ). Thirty-nine ( $97.5 \%$ ) of 40 patients had sCD40L in excess of 2 SD above the control mean (Fig. 1a). Plasma sCD40L was also increased in the group of type 1 diabetic patients as compared with their matched control subjects $(8.36 \pm 2.59 \mathrm{vs}$
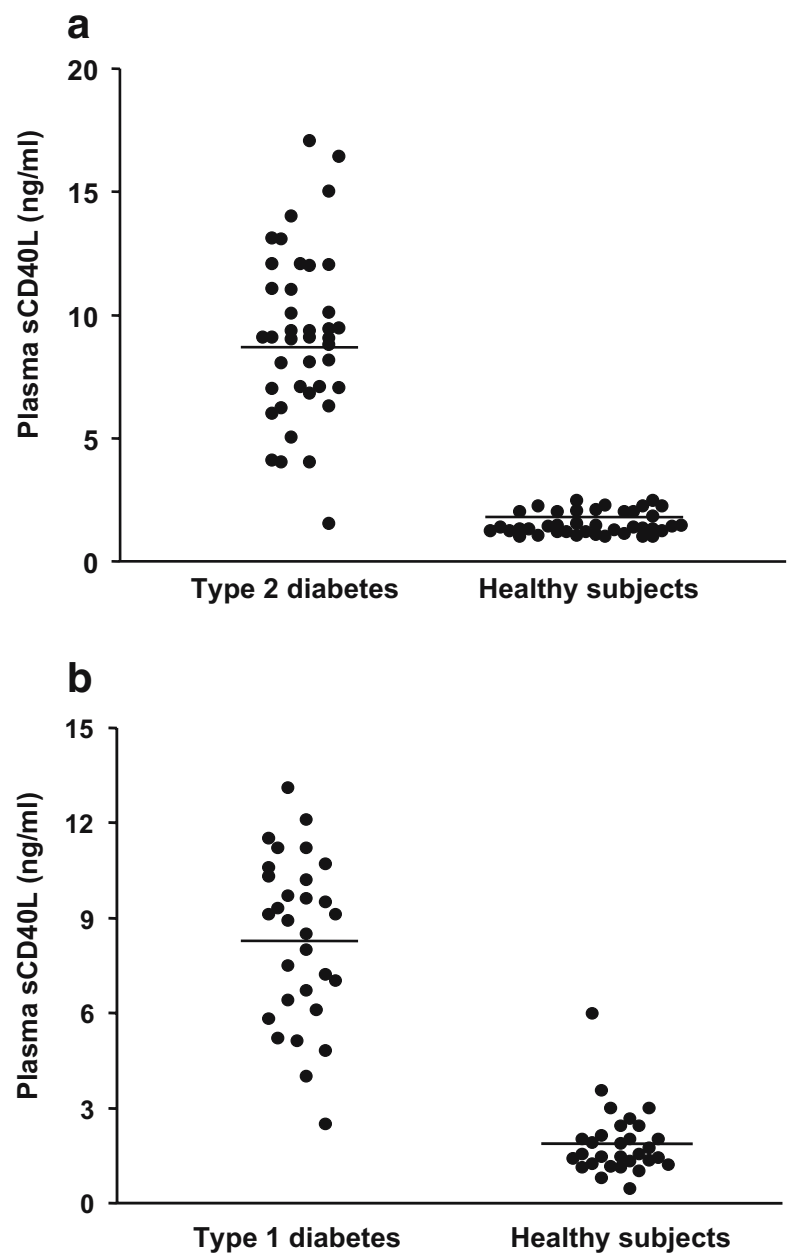

Fig. 1 Plasma levels of sCD40L in patients with type 2 diabetes $(n=40)(\mathbf{a})$ and type 1 diabetes $(n=30)(\mathbf{b})$ vs healthy subjects $(n=40$ and $n=30$, respectively). Dots represent individual measurements; horizontal bars represent mean values for each group. $p<0.0001$ (a); $p<0.0001$ (b)
$1.87 \pm 1 \mathrm{ng} / \mathrm{ml}$, means $\pm \mathrm{SD}, n=30 ; p<0.003$; Fig. $1 \mathrm{~b})$. All but one $(96.6 \%)$ of the 30 patients had SCD40L in excess of 2 SD above the control mean (Fig. 1b). Finally, no significant differences in plasma sCD40L were observed between patients with type 2 diabetes and patients with type 1 diabetes. Similar results were also observed when sCD40L was measured in serum $(9.23 \pm 3.9$ vs $1.92 \pm 0.8$ and $9.3 \pm 2.6$ vs $2 \pm$ $1.1 \mathrm{ng} / \mathrm{ml}$, respectively for type 2 and type 1 diabetes, means \pm SD $p<0.0001)$. Thus, a significant correlation $\left(R^{2}=0.825\right)$ was observed between plasma and serum sCD40L determinations. These data seem to suggest that, at least in diabetic patients, sCD40L measured in serum reflects circulating SCD40L rather than production of SCD40L from platelets activated during serum preparation.

In vivo biosynthesis of adhesion molecules and MCP-1 in diabetic patients

Endothelial perturbation was significantly higher in patients with diabetes (Fig. 2). In fact, both type 2 and type 1 diabetic patients showed higher $(p<0.0001)$ levels of sICAM-1 $(598 \pm 32$ vs $367 \pm 29$ and $511 \pm 18$ vs $314 \pm 23 \mathrm{ng} / \mathrm{ml}$, respectively), sVCAM-1 (1921 \pm 97 vs $943 \pm 64$ and $1874 \pm 61$ vs $1011 \pm 51 \mathrm{ng} / \mathrm{ml}$, respectively) and, sE-selectin (61 \pm 6 vs $38 \pm 4$ and $64 \pm 4$ vs $37 \pm 3 \mathrm{ng} / \mathrm{ml}$, respectively) as compared with control subjects (Fig. 2). Accordingly, both in type 2 and in type 1 diabetic patients we found a positive ( $p<$ 0.0001 ) correlation between circulating SCD40L and circulating sICAM- $1\left(R^{2}=0.522\right.$ and $\left.R^{2}=0.492\right)$, sVCAM- 1 $\left(R^{2}=0.481\right.$ and $\left.R^{2}=0.536\right)$, and sE-selectin $\left(R^{2}=0.512\right.$ and $R^{2}=0.532$ ).

Similarly, MCP-1 levels were significantly $(p<0.0001)$ higher both in type 2 and in type 1 diabetic patients with respect to controls $(98 \pm 11$ vs $57 \pm 6$ and $87 \pm 8$ vs $51 \pm 5 \mathrm{pg} / \mathrm{ml}$, respectively), and a positive correlation $(p<0.0001)$ was found between circulating $\mathrm{SCD} 40 \mathrm{~L}$ and circulating MCP-1 $\left(R^{2}=0.543\right.$ and $\left.R^{2}=0.421\right)$.

\section{Effect of SCD40L on endothelial activation in vitro}

To further examine whether the higher grade of endothelial activation observed in diabetic patients was a direct consequence of enhanced circulating SCD40L, we next studied the effect of serum collected from diabetic patients on the generation of sICAM-1 and MCP-1 in ECs in vitro (Fig. 3). ECs spontaneously generated considerable levels of sICAM1 and MCP-1 when cultured with serum collected from patients with both type 2 diabetes ( $623 \pm 72$ vs $248 \pm 22$ and $24.2 \pm 2$ vs $10.3 \pm 2 \mathrm{ng} / \mathrm{ml}$, respectively; Fig. $3 ; p<0.0001)$ and type 1 diabetes $(711 \pm 77$ vs $259 \pm 13$ and $22 \pm 2$ vs $9 \pm 1 \mathrm{ng} /$ $\mathrm{ml}$, respectively; $p<0.0001$ ), whereas no significant generation was measured in cells cultured with serum from the controls (Fig. 3). The stronger stimulatory effect of sCD40Lrich serum from diabetic patients was replicated by exogenous sCD40L $(10 \mathrm{ng} / \mathrm{ml})$, while it was blocked by a blocking monoclonal antibody for CD40L (Fig. 3), thus confirming the causal role of $\mathrm{sCD} 40 \mathrm{~L}$ in sICAM-1 and 
SICAM-1

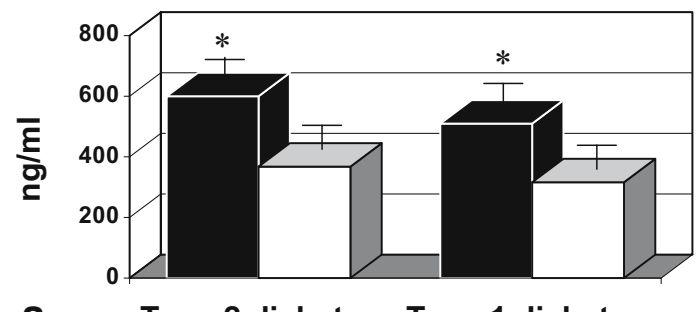

a

Type 2 diabetes Type 1 diabetes

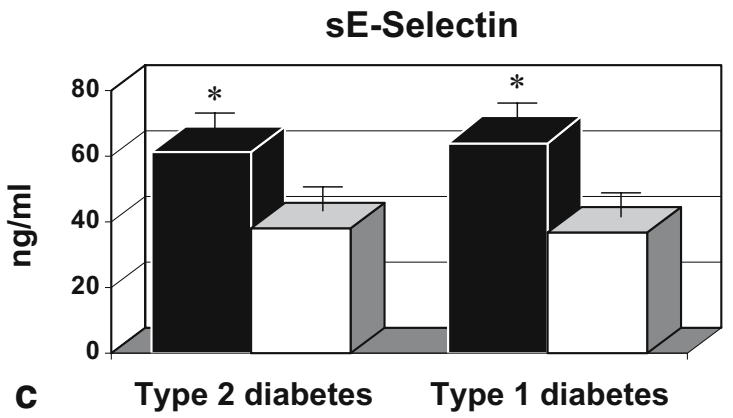

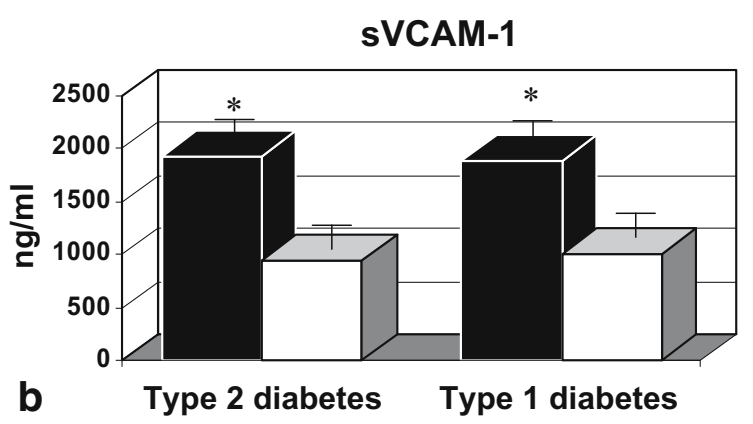

MCP-1

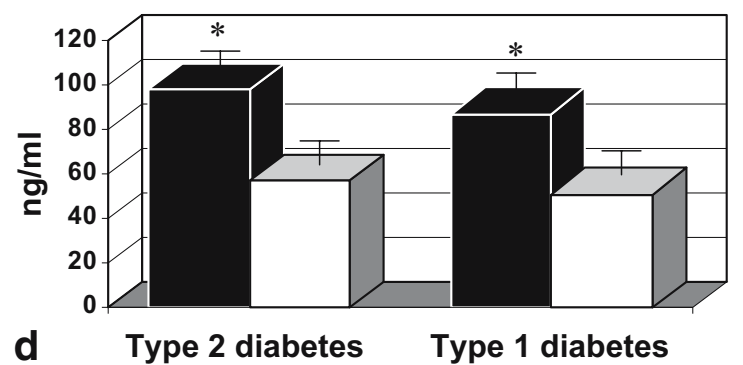

Fig. 2 Plasma levels of sICAM-1 (a), sVCAM-1 (b), sE-selectin (c) and MCP-1 (d) measured in patients with type 2 and type 1 diabetes (black bar) and in matched healthy controls (open bar). Bars and vertical lines represent means $\pm \mathrm{SD} .{ }^{*} p<0.0001$ vs healthy subjects

MCP-1 generation in ECs. The high purity of recombinant $\operatorname{sCD} 40 \mathrm{~L}(>95 \%)$ and the low endotoxin content $(<0.01 \mathrm{ng}$ endotoxin/ $\mu \mathrm{g}$ purified protein) excluded that the effect of recombinant $\mathrm{SCD} 40 \mathrm{~L}$ was due to contaminating endotoxin.

The incubation of cells with serum from healthy subjects containing anti-sCD40L monoclonal antibody excluded any non-specific effect of this antibody on cell function. Furthermore, the isotype-matched control antibody had no effect on SICAM-1 and MCP-1 generation.

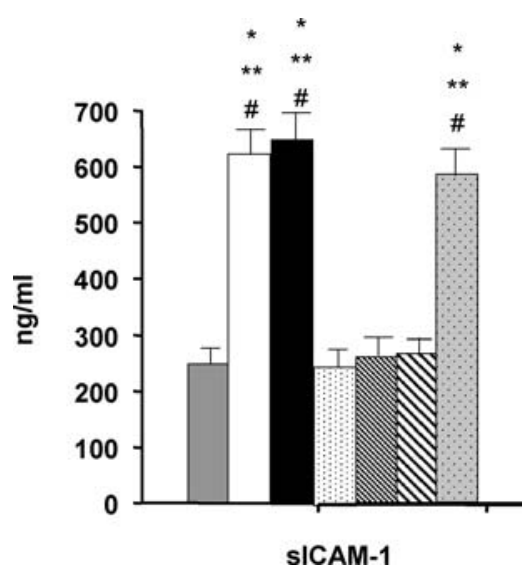

Fig. 3 Effect of serum from type 2 diabetic patients on spontaneous generation of sICAM-1 and MCP-1 in ECs after $3 \mathrm{~h}$ in culture. Cells were incubated with serum from healthy subjects (HS) (grey bar, $n=40$ ), type 2 diabetes (T2DM) (open bar, $n=40$ ), HS+exogenous SCD40L (black bar, $n=40$ ), HS+anti-sCD40L (stippled bar, $n=40$ ), HS+sCD40L+anti-sCD40L (finely hatched bar, $n=40$ ), T2DM plus

\section{Effect of sCD40L on EC migration in vitro}

Migration (per cent of control, Fig. 4) was significantly $(p<0.001)$ abrogated in ECs incubated for 3 or $18 \mathrm{~h}$ with serum from patients with type 2 diabetes and high sCD40L levels $(-45.1 \pm 2.1$ and $-73.2 \pm 1.9 \%$, respectively). In contrast, no significant inhibition was measured in ECs cultured with serum from matched control subjects with low sCD40L levels ( $-10 \pm 1.8$ and $-14 \pm 4.3 \%$, respectively). Im- 
Fig. 4 Effect of serum from type 2 diabetic patients on EC migration after $3 \mathrm{~h}$ (open bar) or $18 \mathrm{~h}$ (black bar) of incubation. Note the inhibitory effect of blocking monoclonal antibody for CD40L. Bars and vertical lines represent means $\pm \mathrm{SD} .{ }^{*} p<0.001$ vs healthy subjects; $* * p<0.001$ vs type 2 diabetes + anti-sCD $40 \mathrm{~L}$

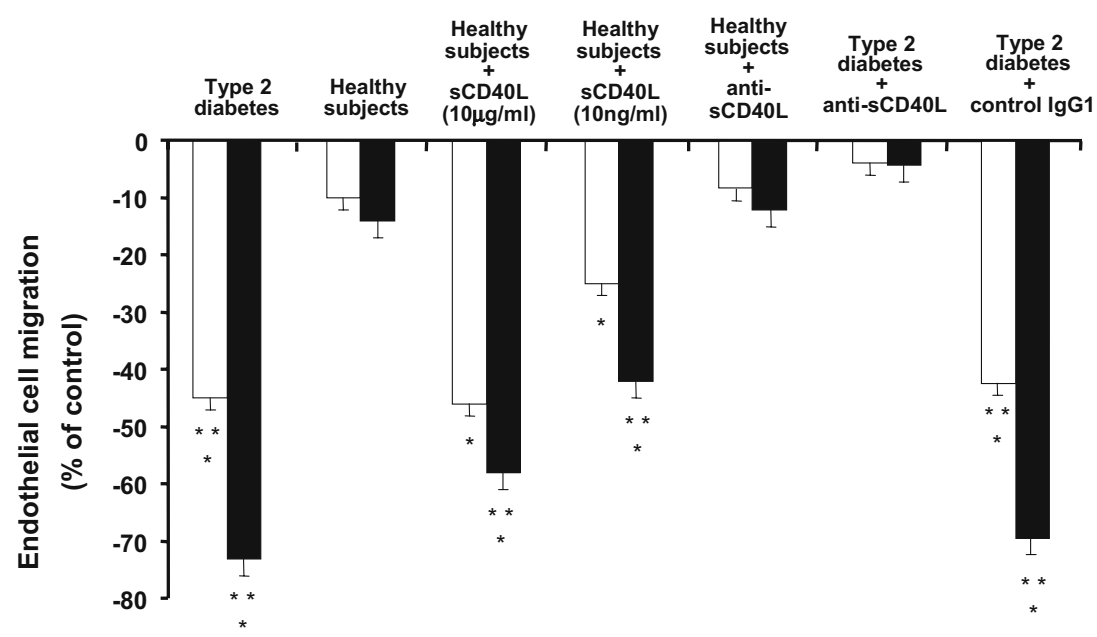

portantly, the stronger inhibitory effect of serum from diabetic patients was replicated by exogenous SCD40L (for 10 $\mu \mathrm{g} / \mathrm{ml}:-46 \pm 2$ and $-58.3 \pm 3 \%$, respectively, $p<0.001$; for 10 $\mathrm{ng} / \mathrm{ml}:-25 \pm 1$ and $-42 \pm 2 \%$, respectively, $p<0.001$ ), while it was completely reversed by neutralising antibody against CD40L ( $-4 \pm 2.4$ and $-4.3 \pm 3.2 \%$, respectively). The antiSCD40L monoclonal antibody had no effect on EC vitality as evaluated by the MTS colorimetric assay. Moreover, the incubation of ECs with serum from healthy subjects and antisCD40L monoclonal antibody excluded any non-specific effect of this antibody on cell function. Furthermore, no effect of isotype-matched control antibody on endothelial migration was found.

Notably, the antibody alone had no effect on EC migration $(-1.2 \pm 2.8 \%$ after $18 \mathrm{~h}, p=\mathrm{NS}$ vs medium alone), thus ruling out the hypothesis of any non-specific effect of the antibody per se. Similarly, the presence in culture medium of sCD40/anti-sCD40L complexes generated in antibody excess (1:5) did not significantly affect EC migratory ability $(-1.0 \pm 1.9 \%$ after $18 \mathrm{~h}, p=\mathrm{NS}$ vs medium alone), thus demonstrating that complexes of antibody and protein have direct effects on cell motility. Finally, the presence of the anti-oxidant $N$-acetyl-cysteine strongly antagonised the effect of $\mathrm{SCD} 40 \mathrm{~L}$ on EC migration, thus confirming the key role of oxidant stress in the pathophysiology of SCD40Ldependent endothelial dysfunction.

Effect of sCD40L on monocyte inflammatory status in vitro

To examine the relationship between circulating sCD40L and monocyte activity in diabetic patients, healthy monocytes were evaluated for spontaneous $\mathrm{O}_{2}^{-}$generation after culturing with either serum from the 70 diabetic patients with broad-spectrum sCD40L levels (range 1.9-17.9 ng/ $\mathrm{ml})$, or serum from ten controls $(<1.1 \mathrm{ng} / \mathrm{ml})$. Monocytes spontaneously generated considerable levels of $\mathrm{O}_{2}^{-}$when cultured with serum collected from both type 2 diabetic patients $\left(11.3 \pm 2 \mathrm{nmol} \cdot 60 \mathrm{~min}^{-1} \cdot 10^{6}\right.$ cells $\left.^{-1}, p<0.0001\right)$ and type 1 diabetic patients $\left(12.3 \pm 1.9 \mathrm{nmol} \cdot 60 \mathrm{~min}^{-1} \cdot 10^{6}\right.$ cells $^{-1}$, $p<0.0001$ ) (Fig. 5). In contrast, no detectable spontaneous $\mathrm{O}_{2}^{-}$generation was observed in monocytes cultured with serum from controls (Fig. 5). The stronger stimulatory effect of serum from diabetic patients was replicated by exogenous sCD40L (also at plasma concentrations) (Fig. 5), while both were blocked by the blocking antibody for CD40L (Fig. 5) or chelerythrine, thus confirming the causal role of sCD40L and PKC in enhanced monocyte $\mathrm{O}_{2}^{-}$generation. The anti-sCD40L monoclonal antibody had no effect on cell vitality as evaluated by the MTS colorimetric assay. Moreover, the incubation of cells with serum from healthy subjects containing anti-sCD40L monoclonal antibody excluded any non-specific effect of this antibody on cell function. The isotype-matched control antibody had no effect on

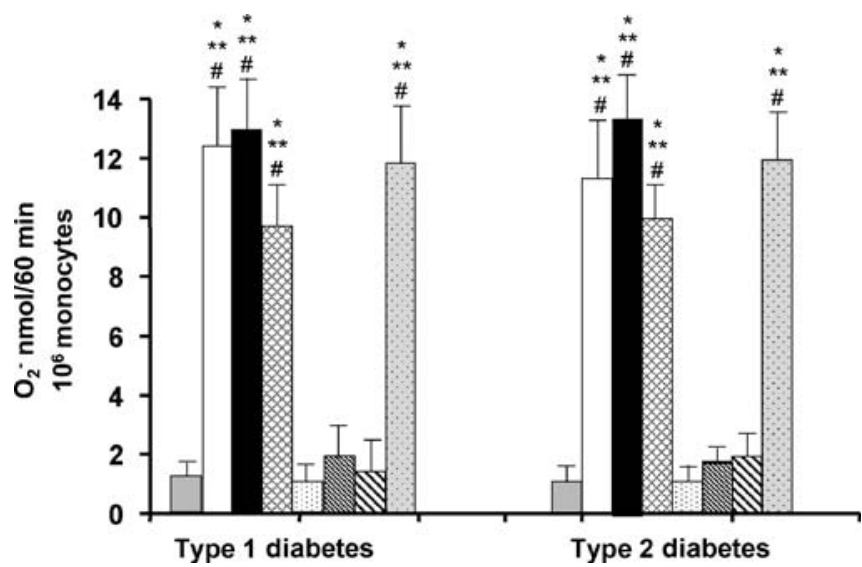

Fig. 5 Effect of serum from type 2 and type 1 diabetic patients on spontaneous generation of $\mathrm{O}_{2}^{-}$in monocytes. Cells were incubated with serum from healthy subjects (HS) $(n=10)$ (grey bar), diabetic patients (open bar, $n=70)$, HS+exogenous sCD40L $(10 \mu \mathrm{g} / \mathrm{ml})($ black bar, $n=10)$, HS+exogenous sCD40L $(10 \mathrm{ng} / \mathrm{ml})$ (cross-hatched bar, $n=10)$, HS+anti-sCD40L (stippled bar, $n=10)$, HS+sCD40L $(10 \mu \mathrm{g}$ ) $\mathrm{ml}$ )+anti-sCD40L (finely hatched bar, $n=10$ ), diabetic patients plus anti-sCD40L (coarsely hatched bar, $n=70$ ), or diabetic patients plus isotype-matched control antibody (stippled grey bar, $n=70$ ). Note the inhibitory effect of blocking monoclonal antibody for CD40L. Bars and vertical lines represent means \pm SD. ${ }^{*} p<0.0001$ vs HS ${ }^{* *} p<0.0001$ vs HS+sCD40L + anti-sCD $40 \mathrm{~L} ;{ }^{\#} p<0.0001$ vs diabetes+ anti-sCD40L 
$\mathrm{O}_{2}^{-}$generation. Accordingly, both in type 2 and in type 1 diabetic patients we found a positive $(p<0.0001)$ correlation $\left(R^{2}=0.523\right.$ and $\left.R^{2}=0.456\right)$ between circulating $\mathrm{SCD} 40 \mathrm{~L}$ and monocyte $\mathrm{O}_{2}^{-}$generation in vitro.

Influence of metabolic control on sCD40L biosynthesis

Twelve patients with type 1 diabetes who had not achieved adequate metabolic control $\left(\mathrm{HbA}_{1} \mathrm{c}>8 \%\right)$ were subjected to intensive monitoring and treatment over 5 years, and blood samples were obtained before and after metabolic control. At the end of this period, $\mathrm{HbA}_{1} \mathrm{c}$ fell from $8.87 \pm 1.3$ to $7.3 \pm$ $1.1 \%(p<0.05)$. Improvement in metabolic control was associated with a statistically significant $(p<0.05) 37.5 \%$ reduction in SCD40L biosynthesis $(10.4 \pm 1.5$ vs $6.5 \pm 1 \mathrm{ng} / \mathrm{ml}$, means \pm SD) (Fig. 6).

Next, we assessed the association between plasma SCD40L and glycaemic control in the two groups of diabetic patients. SCD40L showed a positive correlation with $\mathrm{HbA}_{1} \mathrm{c}$ $(p<0.0001)$ and plasma fasting glucose $(p<0.001)$, both in the 40 patients with type 2 diabetes $\left(R^{2}=0.678\right.$ and $R^{2}=$ 0.421 , respectively) and in the 30 patients with type 1 diabetes $\left(R^{2}=0.622\right.$ and $R^{2}=0.454$, respectively).
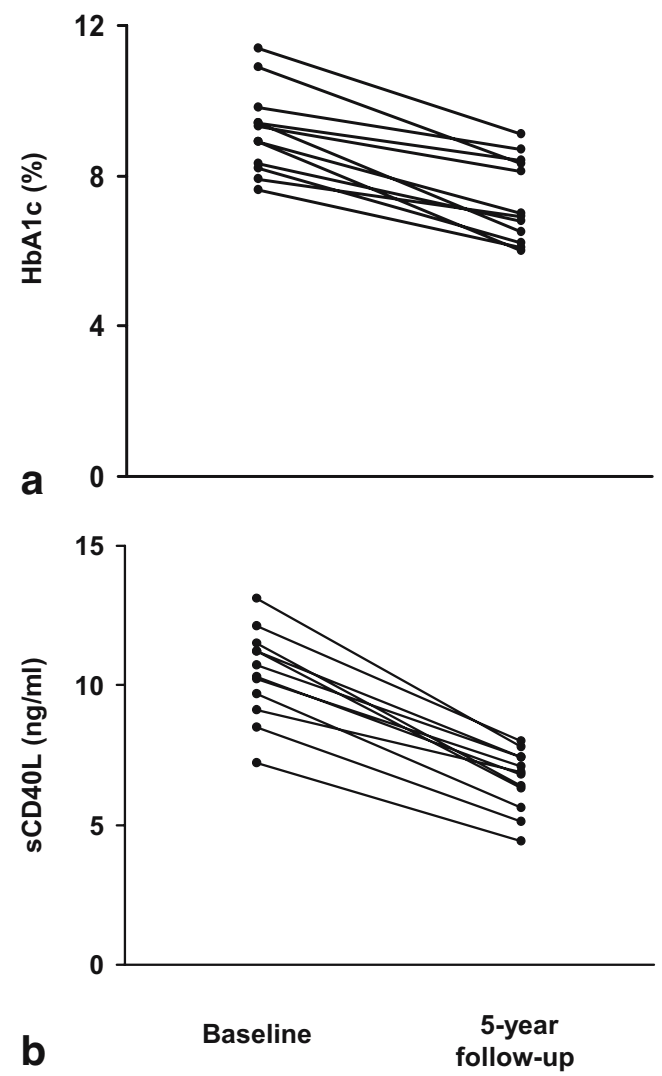

Fig. 6 Effect of improved glycaemic control on plasma SCD40L in 12 patients with type 1 diabetes and poor glycaemic control at baseline. $p<0.05$ (a); $p<0.05$ (b)

\section{Discussion}

Despite evidence of enhanced SCD40L recently reported in patients with diabetes [16, 17], no studies have yet addressed to what extent SCD40L may modulate the complex inflammatory reaction ultimately responsible for the accelerated atherosclerosis seen in diabetes, and whether longterm intensive glycaemic control might diminish circulating SCD40L and related inflammatory state.

Thus, this study: (1) provides in vitro evidence that enhanced $\mathrm{SCD} 40 \mathrm{~L}$ accumulation in diabetic plasma may modulate endothelial and monocyte function in humans; and (2) identifies a strong positive correlation between persistent hyperglycaemia and enhanced SCD40L generation in humans.

In this study, diabetic patients showed higher levels of sCD40L, significantly evident when compared with control subjects. In addition, the increase in $\mathrm{sCD} 40 \mathrm{~L}$ was irrespective of diabetes type, identifying in persistent hyperglycaemia the common pathogenetic element.

The striking effect of sCD40L in increasing the inflammatory reaction in ECs and monocytes raises the question of the origin of this mediator. Platelets are major contributors to enhanced $\mathrm{sCD} 40 \mathrm{~L}$ in patients with acute coronary syndrome [5] and hypercholesterolaemia [6], and studies on the cellular distribution of CD40L indicate that $>95 \%$ of the circulating $\mathrm{SCD} 40 \mathrm{~L}$ exists in platelets [10]. It is important to note that we have previously described in patients with diabetes enhanced thromboxane $A_{2}$ biosynthesis $[20,21]$ and isoprostanes formation [21], mediators that may both induce and amplify platelet activation. Thus, platelets may be a source of localised high concentrations of pro-inflammatory $\mathrm{CD} 40 \mathrm{~L}$, both on the surface and in the immediate environment as they shed SCD40L [10].

Activated platelets have been found to stimulate adhesion molecule and MCP-1 production in ECs through enhanced sCD40L secretion and direct platelet-endothelium contact mediated by CD40L expression on the platelet surface [2]. Such a mechanism has recently been found to be operative in patients with unstable angina [5], and it is conceivable that such a platelet-endothelium interaction may also contribute to the enhanced expression of adhesion molecules and MCP-1 observed in patients with diabetes. In fact, our observation that $\mathrm{SCD} 40 \mathrm{~L}$ is positively correlated with circulating adhesion molecules and MCP-1 in vivo, and may influence the expression of sICAM-1, sVCAM-1, sEselectin and MCP-1 in ECs in vitro, strongly supports this hypothesis.

Importantly, because none of our patients received aspirin throughout the study, we cannot exclude a potential positive effect of aspirin therapy on SCD40L levels in diabetes. Thus, despite recent evidence [7] suggesting that aspirin administration did not alleviate the enhanced risk of future cardiovascular events associated with high sCD40L levels in healthy subjects, additional studies are required to investigate this point in patients with diabetes mellitus.

Alternatively, we cannot exclude that vascular smooth muscle cells, macrophages and lymphocytes may be involved also, at least in part, in sCD40L generation in the setting of diabetes. Thus, a contribution to enhanced SCD40L 
in diabetic patients could be due to inflammatory cells, chronically activated in patients with diabetes.

Thus, our data contribute to the identification of sCD40L as the crucial link between a subclinical inflammatory state at baseline and vessel hyper-responsiveness to injury, ultimately responsible for the accelerated atherosclerosis seen in diabetes. In this light, enhanced SCD40L in diabetic patients is not simply a marker of higher immune activation, but is also actively involved in pathogenetic processes in these patients. Indeed, whatever the cellular source(s), sCD40L may both indirectly and directly have important pathophysiological consequences in patients with diabetes. In fact, CD40L-CD40 interaction may be involved in the process of atherothrombosis through the complex biological effects that it exerts on a number of cell types. First, SCD40L may exercise profound influences on endothelial function. In fact, we demonstrate in this study that sCD40L stimulates the endothelial expression of adhesion molecules critically involved in the early phase of atherogenesis, may activate ECs to release MCP-1, a major signal for inflammatory cell accumulation in the injured vessel [14], and finally exerts a profound inhibitory effect on endothelial migration, a process considered critical for the re-endothelialisation of the injured vessel. EC migration is a tightly regulated process modulated by a complex set of inward and outward signals leading to dynamic and coordinated changes in cytoskeleton organisation and cell adhesion. In particular, adequate endothelium-derived nitric oxide (NO) availability is required for EC migration. In this regard, Urbich et al. [12] have recently demonstrated that sCD40L inhibited EC motility in vitro by reducing NO through an increased generation of ROS. Indeed, the involvement of ROS in the anti-migratory effect of SCD40L has been demonstrated by using anti-oxidant treatments both in the study from Urbich et al. [12] and in our own.

In addition to the effects on ECs, sCD40L may exercise profound influences on monocytes also. In fact, in the present study we found that the raised SCD40L levels in serum from diabetic patients had enhancing effects on spontaneous ROS generation in monocytes. Thus, if this enhancement also occurs in vivo, it may be directly involved in the rapid progression of atherosclerosis. In fact, the generation of ROS has profound and wide-ranging effects that can dramatically increase vascular toxicity and initiate a cascade of molecular and cellular responses. Superoxide anion can inactivate $\mathrm{NO}$, reducing the vasoactive levels of NO and diminishing NO-dependent EC migration [12]. Furthermore, these free radicals with strong cytotoxic potency may cause either direct arterial wall injury or may initiate and/or accelerate secondary processes, including activation of phagocyte-platelet-EC interactions [22], thus further sustaining the detrimental action of platelet-derived sCD40L on ECs. Enhanced $\mathrm{O}_{2}^{-}$generation may also amplify inflammatory gene production. In fact, many vascular biologists now accord a central role to augmented transcription of several atherosclerosis-related genes by the oxidant-sensitive regulatory pathway involving nuclear factor $\mathrm{kB}(\mathrm{NF}-\mathrm{kB})$ [23]. Exposure to extracellular $\mathrm{O}_{2}^{-}$activates the NF- $\mathrm{KB}$ regulatory complex and triggers in ECs and monocytes the transcription of genes that encode certain growth factors, adhesion molecules, chemo-attractant cytokines such as MCP-1, and enzymes that can influence extracellular matrix metabolism. Thus, increased $\mathrm{O}_{2}^{-}$generation in monocytes may enhance the synthesis of MCP-1 in these same cells through an autocrine mechanism [24], possibly representing a vicious circle implicated in accelerated atherosclerosis. Moreover, $\mathrm{O}_{2}^{-}$generation might evoke a secondary adhesion molecule and MCP-1 response from ECs, and establish a positive, self-stimulatory paracrine feedback loop, amplifying and sustaining the proliferative response [23]. Finally, superoxide generation by activated platelets may aggravate endothelial function at the site of platelet aggregation, possibly contributing to acute vessel occlusion. It is also important to consider the fact that superoxide production in monocytes is due to increased NADPH oxidase activity, an enzyme which has been linked strongly in causing endothelial dysfunction in experimental models and in vessels from diabetic patients $[25,26]$. Thus, our data seem to suggest that $\mathrm{SCD} 40 \mathrm{~L}$, in addition to inducing direct inhibition of EC migration, is also involved in the control of the endothelial- and monocyte-mediated inflammatory reactions, ultimately responsible for accelerated atherosclerosis in diabetes.

The fact that impaired glycaemic control, rather than the attendant macrovascular complications, is responsible for enhanced formation of $\mathrm{SCD} 40 \mathrm{~L}$ in diabetes is supported in this study by the absence of manifest coronary, cerebrovascular and peripheral disease in studied type 2 and type 1 diabetic patients. In addition, the active role of $\mathrm{SCD} 40 \mathrm{~L}$ in modulating vascular reactivity has also been demonstrated in this study in vitro by the use of the CD40L-neutralising antibody.

We further examined the relationship between metabolic control and SCD40L formation by studying 12 type 1 diabetic patients with inadequate glycaemic control before and after intensive insulin treatment, and closer monitoring. Reduced blood glucose levels were associated with a fall in plasma sCD40L biosynthesis rates, the average extent of which showed a remarkably good fit with the linear relationship between blood $\mathrm{HbA}_{1} \mathrm{c}$ and blood $\mathrm{sCD} 40 \mathrm{~L}$, as established in the two groups of diabetic patients under baseline conditions. Improvement of metabolic control in these patients was accompanied by a statistically significant reduction in $\mathrm{SCD} 40 \mathrm{~L}$ biosynthesis by $37.5 \%$. On the basis of these findings, it is tempting to speculate that changes in the rate of sCD40L biosynthesis may represent an important biochemical link between altered glycaemic control, platelet activation and vascular dysfunction in diabetes.

A potential limitation of this study is that we have analysed only the soluble but not the membrane-bound CD40L, despite the fact that cell-surface CD40 and CD40L expression are also relevant components of atherogenesis. However, it should be noted that at this time only sCD40L but not membrane-bound CD40L has been identified as an independent predictor of future cardiovascular events in humans $[7,8]$.

In conclusion, our results are consistent with the hypothesis that upregulation of $\mathrm{SCD} 40 \mathrm{~L}$ in patients with diabe- 
tes results in the activation of ECs and in the recruitment of monocytes and tissue macrophages to the arterial wall, possibly contributing to the accelerated development of atherosclerosis seen in diabetes. Further understanding of the mechanism(s) by which sCD40L is produced and acts might provide insight into therapies to limit the progression of diabetic atherosclerosis.

Acknowledgements We wish to thank Maria Teresa Anzellotti for assistance in clinical studies.

\section{References}

1. Singer DE, Nathan DM, Anderson KM, Wilson PW, Evans JC (1992) Association of $\mathrm{HbA}_{1} \mathrm{c}$ with prevalent cardiovascular disease in the original cohort of the Framingham Heart Study. Diabetes 41:202-209

2. Henn V, Slupsky JR, Grafe M et al (1998) CD40 ligand on activated platelets triggers an inflammatory reaction of endothelial cells. Nature 391:591-594

3. Mach F, Schonbeck U, Sukhova GK et al (1997) Functional CD40 ligand is expressed on human vascular endothelial cells, smooth muscle cells, and macrophages: implication for CD40CD40 ligand signalling in atherosclerosis. Proc Natl Acad Sci U S A 94:1931-1936

4. Mach F, Schonbeck U, Sukhova GK, Atkinson E, Libby P (1998) Reduction of atherosclerosis in mice by inhibition of CD40 signalling. Nature 394:200-203

5. Aukrust P, Müller F, Ueland T et al (1999) Enhanced levels of soluble and membrane-bound CD40 ligand in patients with unstable angina. Circulation 100:614-620

6. Cipollone F, Mezzetti A, Porreca E et al (2002) Association between enhanced soluble CD40L and prothrombotic state in hypercholesterolemia: effects of statin therapy. Circulation 106: 399-402

7. Schönbeck U, Varo N, Libby P, Buring J, Ridker PM (2001) Soluble CD40L and cardiovascular risk in women. Circulation 104:2266-2268

8. Heeschen C, Dimmeler S, Hamm CW, CAPTURE Study Investigators et al (2003) Soluble CD40 ligand in acute coronary syndromes. N Engl J Med 348:1104-1111

9. Cipollone F, Ferri C, Desideri G et al (2003) Preprocedural levels of soluble CD40L are predictive of enhanced inflammatory response and restenosis after coronary angioplasty. Circulation 108:2776-2782

10. André P, Nannizzi-Alaimo L, Prasad SK, Phillips DR (2002) Platelet-derived CD40L: the switch-hitting player of cardiovascular disease. Circulation 106:896-899

11. André P, Prasad KS, Denis CV et al (2002) CD40L stabilizes arterial thrombi by a beta3 integrin-dependent mechanism. Nat Med 8:247-252

12. Urbich C, Dernbach E, Aicher A, Zeiher AM, Dimmeler S (2002) CD40 ligand inhibits endothelial cell migration by increasing production of endothelial reactive oxygen species. Circulation 106:981-986
13. Bagg W, Ferri C, Desideri G, Gamble G, Ockelford P, Braatvedt GD (2001) The influences of obesity and glycaemic control on endothelial activation in patients with type 2 diabetes. J Clin Endocrinol Metab 86:5491-5497

14. Cipollone F, Marini M, Fazia M et al (2001) Elevated circulating levels of monocyte chemoattractant protein-1 in patients with restenosis after coronary angioplasty. Arterioscler Thromb Vasc Biol 21:327-334

15. Yasukawa $H$, Imaizumi $T$, Matsuoka $H$, Nakashima $A$, Morimatsu M (1997) Inhibition of intimal hyperplasia after balloon injury by antibodies to intercellular adhesion molecule-1 and lymphocyte function-associated antigen-1. Circulation 95: $1515-1522$

16. Marx N, Imhof A, Froehlich J et al (2003) Effect of rosiglitazone treatment on soluble CD40L in patients with type 2 diabetes and coronary artery disease. Circulation 107:1954-1957

17. Varo N, Vincent D, Libby P et al (2003) Elevated plasma levels of the atherogenic mediator soluble CD40 ligand in diabetic patients: a novel target of thiazolidinediones. Circulation 107: 2664-2669

18. American Diabetes Association (2003) Clinical practice recommendations 2003. Diabetes Care 26(Suppl 1):1-156

19. Desideri G, Ferri C, Bellini C, De Mattia G, Santucci A (1997) Effects of ACE inhibition on spontaneous and insulin-stimulated endothelin-1 secretion. Diabetes 46:81-86

20. Davì G, Catalano I, Averna M et al (1990) Thromboxane biosynthesis and platelet function in type II diabetes mellitus. N Engl J Med 322:1769-1774

21. Davì G, Ciabattoni G, Consoli A et al (1999) In vivo formation of 8-iso-prostaglandin F2 $\alpha$ and platelet activation in diabetes mellitus: effects of improved metabolic control and vitamin $\mathrm{E}$ supplementation. Circulation 99:224-229

22. Blache D (1995) Involvement of hydrogen and lipid peroxides in acute tobacco smoking-induced platelet hyperactivity. Am J Physiol 268:H679-H685

23. Collins T, Read MA, Neish AS, Whitley MZ, Thanos D, Maniatis T (1995) Transcriptional regulation of endothelial cell adhesion molecules: NF-kappa B and cytokine-inducible enhancers. FASEB J 9:899-909

24. Satriano JA, Shuldiner M, Hora K, Xing Y, Shan Z, Schlondorff D (1993) Oxygen radicals as second messengers for expression of the monocyte chemoattractant protein, JE/MCP-1, and the monocyte colony-stimulating factor, CSF-1, in response to tumour necrosis- $\alpha$ and immunoglobulin G: evidence for involvement of reduced nicotinamide adenine dinucleotide phosphate (NADPH)-dependent oxidase. J Clin Invest 92:1564-1571

25. Hink U, Li H, Mollnau H, Oelze M et al (2001) Mechanisms underlying endothelial dysfunction in diabetes mellitus. Circ Res 88:e14-e22

26. Guzik TJ, Mussa S, Gastaldi D et al (2002) Mechanisms of increased vascular super-oxide production in human diabetes mellitus: role of $\mathrm{NAD}(\mathrm{P}) \mathrm{H}$ oxidase and endothelial nitric oxide synthase. Circulation 105:1656-1662 